\title{
Docking Studies reveal Phytochemicals as the long searched anticancer drugs for Breast Cancer
}

\author{
Saba Ferdous \\ Department of Computer Sciences \\ City University London,UK
}

\author{
Muhammad Usman Mirza \\ Center for Research in Molecular Medicine (CRiMM) \\ The University of Lahore,Pakistan
}

\author{
Usman Saeed \\ Department of Genome Oriented Bioinformatics \\ Technical University Munich, Germany
}

\begin{abstract}
Natural products including phytochemicals have been recently proposed as tumor suppressors. In this paper, docking study is presented to use these phytochemicals for their prospective role in cancers including breast and prostate cancer. The most common type of cancer in women all over the world is breast cancer. The breast cells including cancerous breast cells have receptors for binding with estrogen and progesterone to stimulate a growth response. This crucial property has been exploited to investigate binding properties of phytochemicals with these receptors to generate an antagonist response in order to resolve uncontrolled cancerous growth. The most commonly used breast cancer drugs mainly work against the effects of estrogen on these cells. In this context groups of different set of phytochemicals (3-IMG-Glucosinolates, Anthocyanins, Apigenin, Carnosol, Daidzein, Genistein, Isoflavones and Quercetin) were taken and docked into the active site of Human estrogen receptor (PDB ID: 2IOK). In this study, based on molecular docking, potential phytochemicals have successfully been identified which may be used as anticancer drugs against breast cancer. These studies based on binding energy, docking energy, drug likeness and other relevant scores show that Daidzein, Genistein and Quercetin could be the potential lead molecule for the inhibition of signals potent for Human breast cancer and Leu346, Leu384, Leu387, Phe404 and Leu525 are the most important residues for potential drug targets. This paper is the initial step towards a rational design of novel selective and potent Human estrogen inhibitors for the treatment of cancer.
\end{abstract}

\section{Keywords:}

docking, binding energy, phytochemicals, estrogen, drug design

\section{INTRODUCTION}

Breast Cancer, with an incidence of $10.4 \%$ percent is the second most prevailing type of cancer after lung cancer [2]. Normal breast cells and most breast cancer cells have receptors to bind estrogen and progesterone circulating in the blood [8]. These hormones bind to the receptors and generate a growth response in the form of a signal cascade leading to cell proliferation and growth. Their role in cancerous cells cannot be undermined as estrogen and progesterone function with oncogenes and tumor suppressor genes causing the cell to grow out of control [21]. Breast cells that are estrogen and progesterone receptor positive (i.e., ER+ and $\mathrm{PR}+$ ) are more likely to respond hormonal therapy (e.g., Tamoxifen, Raloxifene, Toremifene) and have a better prognosis than cancers that are hormone receptor negative [8]. Tamoxifen $\left(\right.$ Nolvadex ${ }^{\circledR}$ ) is a drug, taken orally as a tablet, which interferes with the activity of estrogen. Some of the most common side effects of Tamoxifen are serious side effects of Tamoxifen include blood clots, strokes, uterine cancer, and cataracts. Raloxifene may cause serious blood clots to form in the legs, lungs, or eyes. Other reactions experienced include leg swelling/pain, trouble breathing, chest pain, vision changes. Therefore, these side effects make these drugs un suitable for use and require studies on a better alternate. Our research was aimed to find suitable natural products with high binding affinity for breast cancer receptors, which could lead to breast cancer treatment [13]. Phytochemicals are proved to be very successful to diminish the possibility of cancer. Therefore, in this study, the effect of phytochemicals have been observed to target the breast cancer. Phytochemicals may be classified into a number of principal groups [3, 4, 16] mainly Flavonoids and Isoflavones. Isoflavones are a large class of compounds found in plants, many of which are weak estrogens. Interestingly, the chemical structure of Isoflavones is similar to estrogen [17]. It is hypothesized that the structural similarity results in competitive binding against estrogen. Depending on the type of estrogen receptor on the cells, Isoflavones may reduce or activate the activity of estrogen. Isoflavones can compete with estrogen for the same receptor sites thereby decreasing the health risks of excess estrogen. Isoflavones block estrogen, a hormone linked to an increased risk of breast and other hormone-dependent cancers [12]. They act rather like Tamoxifen, a drug widely used to treat and prevent breast cancer. In this study, among different phytochemicals which are tested, Genistein, Quercetin and Daidzein showed promising binding with estrogen receptor due to the fact that the structure of estrogen shares striking resemblance with these pyhtochemicals. As shown in Fig. 1, all are polyphenols sharing structural similarity with the principal mammalian estradiol sex hormone. The structures clearly show common features which include the presence of a pair of hydroxyl groups and a phenolic ring, which is essential for binding to the estrogen receptor (ER) subtypes and . In order to determine ER binding ability and transcriptional activation, the hydroxyl group's position are very important with maximal potency achieved at positions four, six and seven [10, 18, 22]. 
<smiles>COc1ccc(-c2c(O)oc3cc(O)c(O)cc3c2=O)cc1O</smiles><smiles></smiles>

Quercetin

Fig. 1. 2D Structure of daidzein, Genistein, Quercetin and Estrogen showing structural similarity.

\section{MATERIALS AND METHODS}

\subsection{Retrieval of protein and ligands from database}

The three dimensional structure of Human estrogen receptor was obtained from the protein data bank (PDB ID: 2IOK, Res: $2.40 \AA$ A $)|7|$ complexed with selective potent inhibitor N-[(1R)-3-(4-HYDROXYPHENYL)-1-METHYLPROPYL]-2(2-PHENYL-1H-INDOL-3-YL)ACETAMIDE and the structure of all the phytochemicals in this study were retrieved from Pubchem compound database [20]. Structural formulas for all the selected drug molecules are given in Fig. 2.

\subsection{Protein and Ligand preparation}

The raw protein from protein data bank with PDB ID 2IOK named Human estrogen receptor is further prepared for docking studies. The protein receptor was initially prepared by removing all the Hetatms and water molecules followed by subsequent energy minimization to remove the bad steric clashes using the software Chimera [15] for 1000 steps at RMS gradient of 0.02 with 10 as update interval and using AMBER [19] ff $12 \mathrm{SB}$ as force field. The 2D structures of molecules were converted to 3D structures with the help of open babel (http: //openbabel.org/wiki/Main_Page) followed by the energy minimization using Hyperchem?s MM+ force field

\subsection{Determination of binding site}

Binding and active sites of proteins are often associated with structural pockets and cavities having high affinity for candidate drugs. The catalytic site of Human estrogen receptor was obtained from the information available in the literature. The catalytic residue further examined with the help of Q-SiteFinder [9] and Computed Atlas of Surface Topography of proteins (CASTp) server [6]. Q-SiteFinder uses the interaction energy between the protein and a simple van der Waals probe to locate energetically favorable binding sites. The knowledge base information from literature and from computer program is in agreement (Details are given Supplementary Table 1 and S Fig. 1 and Fig. 2). CASTp server uses the weighted Delaunay triangulation and the alpha complex for shape measurements. It provides identification and measurements of surface accessible pockets as well as interior inaccessible cavities, for proteins and other molecules. It measures analytically the area and volume of each pocket and cavity, both in solvent accessible surface (SA, Richards? surface) and molecular surface (MS, Connolly?s surface).
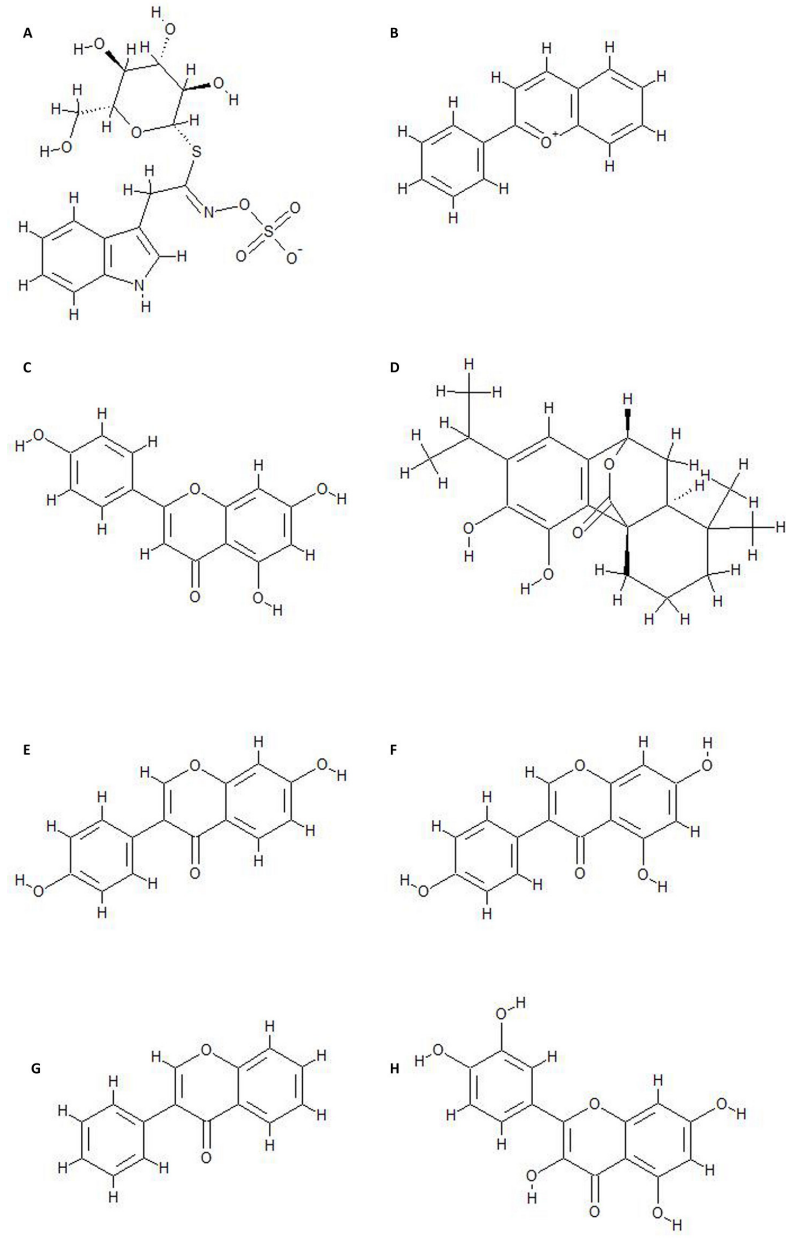

Fig. 2. Structure of compounds used for docking study. (a) 3-IMG-Glucosinolates (b) Anthocyanins (c) Apigenin (d) Carnosol (e) Daidzein (f) Genistein (g) Isoflavones (h) Quercetin

\subsection{Computation of docking score between Human estrogen receptor and phytochemicals}

All computational docking studies were carried out using AUTODOCK 4.0 [14] (version: 1.5.6) installed in a single machine running on a $2.0 \mathrm{GHz}$ Intel core 2 duo processor with $4 \mathrm{~GB}$ RAM and 450 GB hard disk with LINUX (Open Suse) as an operating system. Automated dockings were performed to locate the appropriate binding orientations and conformations of various inhibitors in the Human estrogen receptor binding pocket using AutoDock4.0 tool according to the specified instructions. In brief, polar hydrogen atoms and Kollman charges were assigned to the receptor proteins. For ligands, Gasteiger partial charges were designated and non-polar hydrogen atoms were merged. All torsions for ligands were allowed to rotate during docking procedure. The program AutoGrid was used to generate the grid maps. Each grid was centred at the structure of the corresponding receptor. The grid dimensions were $94 \times 153 \times 145$ $\AA^{3}$ with points separated by $0.375 \AA$. For all ligands, random starting positions, random orientations and torsions were used. The translation, quaternion and torsion steps were taken from default values indicated in AutoDock. The Lamarckian genetic algorithm method was used for minimization using default parameters. The standard docking protocol for rigid and flexible ligand docking consisted of 50 runs, using an initial population of 150 randomly placed individuals, with $2.5 \times 10^{5}$ energy evaluations, a maximum number of 27000 iterations, mutation rate 


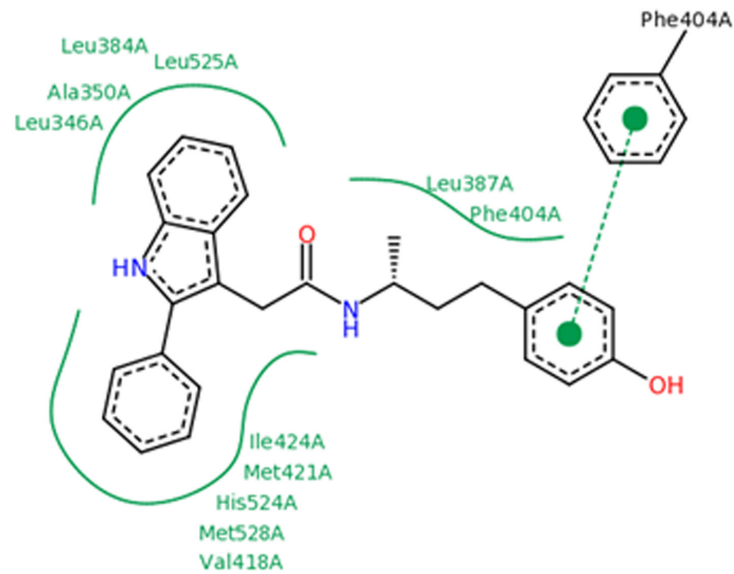

Fig. 3. Binding site in Human estrogen receptor comprised of Leu346, Ala350, Leu384, Leu387, Phe404, Val418, Met421, Ile424, His524 and Leu525

of 0.02 , crossover rate of 0.80 and an elitism value of 1 . Cluster analysis was performed on the docked results using an RMS tolerance of $1.0 \AA$. The binding energy of each cluster is the mean binding energy of all the conformations present within the cluster; the cluster with lowest binding energy and higher number of conformations within it was selected as the docked pose of the particular ligand.

\subsection{Adverse effect prediction}

ADMET (Absorption, Distribution, Metabolism, Excretion and Toxicity) properties were predicted using in-silico methods to know whether the Phytochemicals has the potential of adverse effect in human. In this study, MedChem Designer ${ }^{\mathrm{TM}}[1]$ is used for the calculation of ADMET.

\section{RESULTS AND DISCUSSION}

\subsection{Binding site analysis}

The experimental analysis shows that Leu346, Ala350, Leu384, Leu387, Phe404, Val418, Met421, Ile424, His524 and Leu525 could be the catalytic site residues present in the structure of $\mathrm{Hu}$ man estrogen receptor [7]. Catalytic residues were evaluated by means of various computational tools such as QSiteFinder [9] and CASTp [6]. From the view of Q-SiteFinder, It was observed that catalytic site residues such as Leu346, Ala350, Leu384, Leu 387, Phe404, Val418, Met421 and Ile424 were present in the first predicted site of volume $603 \AA^{3}$. The evidences available suggest that catalytic residues of more than $95 \%$ of the protein were present at least in one of the top three predicted sites when tested using Q-SiteFinder. The program CASTp also supports the results of Q-SiteFinder. The catalytic site residues in the structure of Human estrogen receptor are shown in Fig. 3 . These computational analysis along with experimental fact support that Leu346, Ala350, Leu384, Leu387, Phe404, Val418, Met421, Ile424, His524 and Leu525 act as catalytic residues in the three dimensional structure of Human estrogen receptor [7]. The attained results are in consensus with the above findings.

\subsection{Docking studies of Human estrogen receptor with phytochemicals}

The current investigation showed the behavior of protein ligand complex of Human estrogen receptor with phytochemicals. All the phytochemicals (Fig. 2) included in this study were docked in
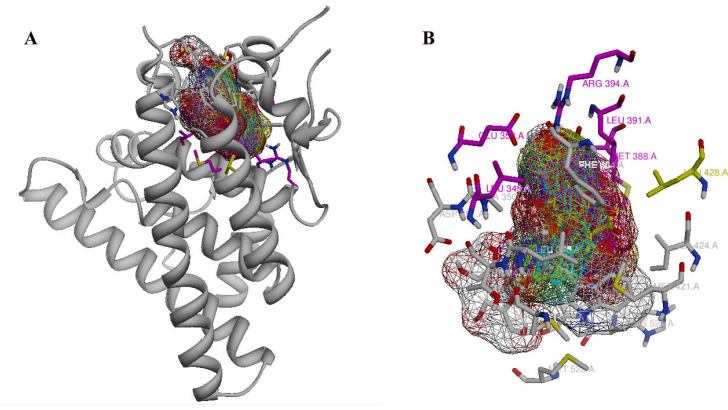

Fig. 4. Binding of Phytochemicals with Human estrogen receptor binding site

the catalytic binding site. The binding site snugly fits the active site cavity making various close contacts with the residues including Leu346, Ala350, Leu384, Leu387, Phe404, Ile424 and Leu525 (Fig 4 and Fig 5). The detailed information of binding residues with all phytochemicals is given in supplementary Table 2 . The current results prove that binding site for human estrogen receptor is conserved for all mentioned phytochemicals and Leu346, Leu384, Leu387, Phe404 and Leu525 are the most important residues for potential drug target. The estimated free energy of binding $(\mathrm{G})$ for the target molecule, Human estrogen receptor with 3-IMG-Glucosinolates, Anthocyanins, Apigenin, Carnosol, Daidzein, Genistein, Isoflavones and Quercetin were found to be $-4.85,-6.56,-5.83,-6.16$, $7.72,-7.62,-7.00$ and $-7.13 \mathrm{kcal} / \mathrm{mol}$ respectively (Table 1 ). It is also observed that Daidzein, Genistein and Quercetin have the better binding affinity with Human estrogen receptor than the other drug molecules. The gradual decrease in G from 3IMG-Glucosinolates to Daidzein may be attributed to the intermolecular interaction energy between the Human estrogen receptor and drug molecules. The number of intermolecular interactions in the docked complexes shown in Table 2 and explanation is given in supplementary data. It shows that number of intermolecular interaction is higher in the case of Daidzein, Genistein and Quercetin compared with other drug molecules (Table 2). This leads to the efficient binding of with Daidzein, Genistein and Quercetin with Human estrogen receptor. Since the binding affinity and docking score is higher, the value of inhibition constant was very less for Daidzein, Genistein and Quercetin. From this observation, it is evident that these three phytochemicals have better binding affinity with the target molecule, Human estrogen receptor, leads to the lesser requirement for the inhibition.

\subsection{Toxicity prediction}

The molecular properties for toxicity analysis of all the phytochemical ligands were calculated and displayed as given in Supplementary Table 3. Ligands with $\mathrm{X} \log P^{3}$ lesser than $5, \log \mathrm{D}$ greater than -4 , molecular weight lesser than 450 , positive value for drug likeness and maximum drug score, possess qualities of less toxic traded drugs. Clearly, these compounds satisfied Lipinski's rule [11] of five and ADMET properties [5].

\section{CONCLUSION}

The protein-ligand interaction plays a significant role in structural based drug designing. In the present work, receptor for Human estrogen has been taken and the potential drugs have been identified that can be used against Breast Cancer. By applying computational approaches, it has been tried to understand the mechanism of interactions and binding affinity between phytochemicals and Human estrogen receptor. The phytochemicals which were used in this study showed the binding energies in the range -7.72 and $-4.85 \mathrm{kcal} / \mathrm{mol}$ and docking energy in the 
Table 1. Autodock estimated free energies of binding (G) of compound a-h in the active site Human Estrogen receptor

\begin{tabular}{lllll}
\hline Ligands & $\begin{array}{l}\text { Binding energy } \\
\mathrm{G}(\mathrm{kcal} / \mathrm{mol})\end{array}$ & $\begin{array}{l}\text { Intermol Energy } \\
(\mathrm{kcal} / \mathrm{mol})\end{array}$ & $\begin{array}{l}\text { Docking Energy } \\
(\mathrm{kcal} / \mathrm{mol})\end{array}$ & $\begin{array}{l}\text { Inhibition } \\
\text { Constant(uM) }\end{array}$ \\
\hline 3-IMG-Glucosinolates & -4.85 & -8.43 & -9.38 & 298.52 \\
\hline Anthocyanins & -6.56 & -6.85 & -7.07 & 10.94 \\
\hline Apigenin & -5.83 & -7.03 & -7.76 & 53.13 \\
\hline Carnosol & -6.16 & -7.06 & -7.6 & 33.62 \\
\hline Daidzein & -7.72 & -8.61 & -8.82 & 2.21 \\
\hline Genistein & -7.62 & -8.81 & -9.62 & 2.69 \\
\hline Isoflavones & -7.00 & -7.3 & -7.55 & 6.73 \\
\hline Quercetin & -7.13 & -8.92 & -10.23 & 5.55 \\
\hline
\end{tabular}

Table 2. Phytochemicals displaying different types of interactions with Human estrogen receptor

\begin{tabular}{|c|c|c|c|c|c|c|}
\hline Ligands & $\begin{array}{l}\text { Number of } \\
\mathrm{H} \text { - bond } \\
\text { interactions }\end{array}$ & $\begin{array}{l}\text { Number of } \\
\text { polar } \\
\text { interactions }\end{array}$ & $\begin{array}{l}\text { Number of } \\
\text { Non-polar } \\
\text { interactions }\end{array}$ & $\begin{array}{l}\text { Number of } \\
\text { hydrophobic } \\
\text { interactions }\end{array}$ & $\begin{array}{l}\text { Number of } \\
\text { - Sigma } \\
\text { interactions }\end{array}$ & $\begin{array}{l}\text { Total Number } \\
\text { of interactions }\end{array}$ \\
\hline 3-IMG-Glucosinolates & 2 & 8 & 5 & 13 & 0 & 28 \\
\hline Anthocyanins & 0 & 0 & 12 & 11 & 1 & 24 \\
\hline Apigenin & 4 & 7 & 5 & 10 & 1 & 27 \\
\hline Carnosol & 1 & 3 & 13 & 10 & 1 & 28 \\
\hline Daidzein & 3 & 7 & 5 & 13 & 0 & 28 \\
\hline Genistein & 3 & 8 & 6 & 13 & 0 & 30 \\
\hline Isoflavones & 1 & 2 & 9 & 11 & 2 & 25 \\
\hline Quercetin & 3 & 5 & 8 & 11 & 1 & 28 \\
\hline
\end{tabular}

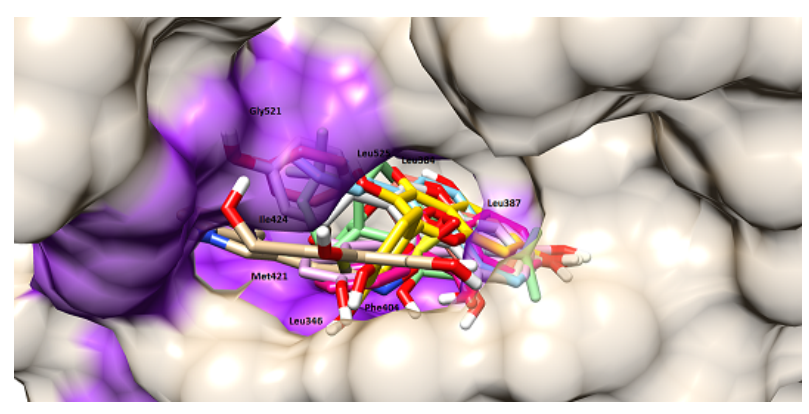

Fig. 5. Phytochemicals showing their binding affinity with estrogen receptor binding site

range -9.62 and $-7.07 \mathrm{kcal} / \mathrm{mol}$ which is in very good agreement with the standard and ideal binding energy. The present analysis also shows that Daidzein, Genistein and Quercetin could be the potential lead molecule for the inhibition of Human estrogen receptor and Leu346, Leu384, Leu387, Phe404 and Leu525 are the most important residues for potential drug target. Hence these natural compounds could be used as the template for designing therapeutic lead molecules which could results into massive reductions in therapeutics development time. This study may be the subject of experimental validation and clinical trials to establish these phytochemicals as more potent drug for the treatment of different cancers in general and breast cancer in particular. In future the ADME/T (Absorption, Distribution, Metabolism, Excretion/Toxicity) properties of these compounds can be calculated using the commercial ADME/T tools available thus reducing the time and cost in drug discovery process. These results will be decisive factor for determining a lead phytochemical for further drug discovery process.

\section{Acknowledgments}

We would like to specially thank Gregory Slabaugh for useful suggestions during the project. There is no funding claim asso- ciated with this project. The work was undertaken by the authors utilizing personal resources available at respective institutes.

\section{Appendix A. Supplementary data}

Supplementary data associated with this article can be found in the online version at, http://histonesolutions.com/ docs/Supplementary\%20material.docx

\section{REFERENCES}

[1] Medchem designer. http://www.simulations-plus . com/Products . aspx?grpID=1\&cID=20\&pID=25 Accessed: 19/02/2013.

[2] World health organization international agency for research on cancer. World Cancer Report, June 2008.

[3] Herman Adlercreutz. Phytoestrogens and breast cancer. The Journal of steroid biochemistry and molecular biology, 83(1):113-118, 2002.

[4] Aedin Cassidy, Bryan Hanley, and Rosa M LamuelaRaventos. Isoflavones, lignans and stilbenes-origins, metabolism and potential importance to human health. Journal of the Science of Food and Agriculture, 80(7):1044-1062, 2000.

[5] John C Dearden. In silico prediction of admet properties: how far have we come? 2007.

[6] Joe Dundas, Zheng Ouyang, Jeffery Tseng, Andrew Binkowski, Yaron Turpaz, and Jie Liang. Castp: computed atlas of surface topography of proteins with structural and topographical mapping of functionally annotated residues. Nucleic acids research, 34(suppl 2):W116-W118, 2006.

[7] K. D. Dykstra, L. Guo, E. T. Birzin, W. Chan, Y. T. Yang, E. C. Hayes, C. A. DaSilva, L. Y. Pai, R. T. Mosley, B. Kraker, P. M. Fitzgerald, F. DiNinno, S. P. Rohrer, J. M. Schaeffer, and M. L. Hammond. Estrogen receptor ligands. Part 16: 2-Aryl indoles as highly subtype selective ligands for ERalpha. Bioorg. Med. Chem. Lett., 17(8):2322-2328, Apr 2007. 
[8] Mark A Espeland, Sally A Shumaker, Marian Limacher, Stephen R Rapp, Therese B Bevers, David H Barad, Laura H Coker, Sarah A Gaussoin, Marcia L Stefanick, Dorothy S Lane, et al. Relative effects of tamoxifen, raloxifene, and conjugated equine estrogens on cognition. Journal of Women's Health, 19(3):371-379, 2010.

[9] Alasdair TR Laurie and Richard M Jackson. Q-sitefinder: an energy-based method for the prediction of proteinligand binding sites. Bioinformatics, 21(9):1908-1916, 2005.

[10] J. C. Le Bail, Y. Champavier, A. J. Chulia, and G. Habrioux. Effects of phytoestrogens on aromatase, 3beta and 17beta-hydroxysteroid dehydrogenase activities and human breast cancer cells. Life Sci., 66(14):1281-1291, Feb 2000.

[11] Christopher A Lipinski, Franco Lombardo, Beryl W Dominy, and Paul J Feeney. Experimental and computational approaches to estimate solubility and permeability in drug discovery and development settings. Advanced drug delivery reviews, 2012.

[12] Roy Ackermann Michelle Harvie. Genesis breast cancer prevention diet. pages 113-114, 2006.

[13] Shoko Morishima, Masa-Aki Shibata, Masahide Ohmichi, and Yoshinori Otsuki. Raloxifene, a selective estrogen receptor modulator, induces mitochondria-mediated apoptosis in human endometrial carcinoma cells. Medical molecular morphology, 41(3):132-138, 2008.

[14] Garrett M Morris, Ruth Huey, William Lindstrom, Michel F Sanner, Richard K Belew, David S Goodsell, and Arthur J Olson. Autodock4 and autodocktools4: Automated docking with selective receptor flexibility. Journal of computational chemistry, 30(16):2785-2791, 2009.

[15] Eric F Pettersen, Thomas D Goddard, Conrad C Huang, Gregory S Couch, Daniel M Greenblatt, Elaine C Meng, and Thomas E Ferrin. Ucsf chimeraa visualization system for exploratory research and analysis. Journal of computational chemistry, 25(13):1605-1612, 2004.

[16] Maria Russo, Carmela Spagnuolo, Idolo Tedesco, and Gian Luigi Russo. Phytochemicals in cancer prevention and therapy: Truth or dare? Toxins, 2(4):517-551, 2010.

[17] Kenneth DR Setchell and Aedin Cassidy. Dietary isoflavones: biological effects and relevance to human health. The journal of nutrition, 129(3):758S-767S, 1999.

[18] Snait Tamir, Mark Eizenberg, Dalia Somjen, Naftali Stern, Rayah Shelach, Alvin Kaye, and Jacob Vaya. Estrogenic and antiproliferative properties of glabridin from licorice in human breast cancer cells. Cancer research, 60(20):5704$5709,2000$.

[19] Junmei Wang, Wei Wang, Peter A Kollman, and David A Case. Automatic atom type and bond type perception in molecular mechanical calculations. Journal of molecular graphics and modelling, 25(2):247-260, 2006.

[20] Yanli Wang, Evan Bolton, Svetlana Dracheva, Karen Karapetyan, Benjamin A Shoemaker, Tugba O Suzek, Jiyao Wang, Jewen Xiao, Jian Zhang, and Stephen H Bryant. An overview of the pubchem bioassay resource. Nucleic acids research, 38(suppl 1):D255-D266, 2010.

[21] Caroline J Witton, Jonathan R Reeves, James J Going, Timothy G Cooke, and John Bartlett. Expression of the her1-4 family of receptor tyrosine kinases in breast cancer. The Journal of pathology, 200(3):290-297, 2003.

[22] Rachel S Rosenberg Zand, David JA Jenkins, and Eleftherios P Diamandis. Steroid hormone activity of flavonoids and related compounds. Breast cancer research and treatment, 62(1):35-49, 2000. 